\title{
Improved extrapolation operator design with the WLSQ method
}

Jan Thorbecke* and Kees Wapenaar, Delft University of Technology, Department of Applied Earth Sciences, The Netherlands

\section{Summary}

In Thorbecke and Rietveld (1994) a weighted least squares technique has been introduced to estimate short, stable and accurate extrapolation operators. This technique produced results which were comparable with the results of Holberg (1988); Blacquiere et al. (1989); Hale (1991); Nautiyal et al. (1993). In this abstract the weighted least squares technique is improved by defining different object functions. 2D Zero-offset migration impulse responses for different types of operators are shown and the Sigsbee2A data set is used to illustrate the accuracy of the extrapolation operators in pre-stack depth migration.

\section{Introduction}

Recursive wavefield extrapolation in the frequency domain extrapolates data from depth level $z_{m}$ to level $z_{m+1}$, where $\Delta z=\left|z_{m+1}-z_{m}\right|$ is small compared to the operator length. Due to the recursive use of the operators special care must be taken about the amplitudes. An amplitude larger than one can lead to unstable extrapolation results, while an amplitude smaller than one will attenuate the wavefield during extrapolation.

In the $k_{x}-\omega$ domain the extrapolation operator for a 2 -dimensional medium is given by equation (1)

$$
\tilde{W}\left(k_{x}, \omega, \Delta z\right)=\exp \left(-j k_{z} \Delta z\right),
$$

with $k_{z}=\sqrt{k^{2}-k_{x}^{2}}, k=\frac{\omega}{c}, \omega$ the angular frequency and $c$ the propagation velocity. Transforming equation (1) back to the spatial domain and truncating it to a shorter operator gives amplitude ripples higher than 1 in the wavenumber domain. It also limits the maximum propagation angle of the extrapolated wavefield. The ideal spatial extrapolation operator would propagate all waves accurately, stable and also consist of a limited number of points for efficiency.

\section{Weighted Least Squares}

The goal in the optimization procedure is to obtain a short spatial convolution operator, which has a wavenumber spectrum over a desired wavenumber band, equal or close to the exact formulation in the frequencywavenumber domain. This problem can be written as the integral equation

$$
\tilde{W}\left(k_{x}\right)=\int_{-x_{1}}^{x_{1}} \exp \left(j k_{x} x\right) W(x) \mathrm{d} x \quad \text { for } \quad\left\|k_{x}\right\| \leq k_{N},
$$

where $W(x)$ is the (unknown) convolution operator. In this integral equation the integration is carried out over a limited spatial interval, representing the short operator. Also the frequency-wavenumber domain of the operator is band limited. The discrete counterpart of this integral is given by

$$
\tilde{\boldsymbol{W}}=\Gamma \boldsymbol{W}
$$

The weighted least squares (WLSQ) solution of equation (3) is given by

$$
\boldsymbol{W}_{o p t}=\left[\boldsymbol{\Gamma}^{H} \tilde{\Lambda} \boldsymbol{\Gamma}\right]^{-1} \boldsymbol{\Gamma}^{H} \tilde{\boldsymbol{\Lambda}} \tilde{\boldsymbol{W}} .
$$

$\boldsymbol{\Gamma}^{H} \tilde{\boldsymbol{\Lambda}} \boldsymbol{\Gamma}$ is a square $M \times M$ matrix, which has to be inverted. For 1-dimensional operators this matrix has a Toeplitz structure and can be inverted efficiently using the Levinson scheme. If in equation (4) the weight matrix is chosen identical to the unit matrix $\boldsymbol{\Lambda}=\mathbf{I}$, then the right hand side of equation (4) is an inverse Fourier transform of N-points, which is truncated to M-points in the spatial domain. In this specific case no optimization is carried out.

By choosing a certain weight function it is possible to reduce the accuracy in the evanescent part $\left(\left\|k_{x}\right\|>k\right)$ and improve the accuracy in the propagating part $\left(\left\|k_{x}\right\| \leq k\right)$ of the extrapolation operator. The recursive extrapolation scheme only demands a stable (amplitude $<1$ ) behavior of the wavenumbers in the evanescent part, the phase in this part is then of no importance. The used weight function is chosen to be a simple block function with a weight of 1 inside the domain of interest (propagating) and a small value (1e-5) outside this band.

For accurate extrapolation results the desired operator $\tilde{\boldsymbol{W}}$ must be equal to the phase-shift operator for the propagating waves, however the behavior outside this part can differ from the phase-shift operator. A so-called smooth operator has been designed in such a way that outside the band of interest the amplitude and the phase are defined by a cubic spline, which goes smoothly to zero:

$$
\begin{gathered}
\left\|\tilde{W}\left(k_{x}, \omega, \Delta z, \alpha\right)\right\|= \begin{cases}1.0 & \left|k_{x}\right| \leq k \sin (\alpha) \\
\text { spline } & \left|k_{x}\right|>k \sin (\alpha), \\
0 & \left|k_{x}\right|=\frac{\pi}{\Delta x}\end{cases} \\
\arg \left(\tilde{W}\left(k_{x}, \omega, \Delta z, \alpha\right)\right)= \begin{cases}-j k_{z} \Delta z & \left|k_{x}\right| \leq k \sin (\alpha) \\
\text { spline } & \left|k_{x}\right|>k \sin (\alpha), \\
0 & \left|k_{x}\right|=\frac{\pi}{\Delta x}\end{cases}
\end{gathered}
$$

where $\alpha$ is the maximum propagation angle of interest. The weight function is still box-shaped. By using this smoother objective function the least-squares algorithm can find a smoother solution and will have less wild oscillation in the wavenumber spectrum. 


\section{Improved extrapolation operator design with the WLSQ method}

The WLSQ optimized convolution operator, based on an object function equal to the phase-shift operator, is shown in Figure 1a. The wavenumber spectrum is stable for all wavenumbers and is accurate within the band of interest. The accuracy of this operator is the same as the operators of Holberg (1988); Blacquiere et al. (1989). Figure 1b shows the WLSQ optimized operator with the smooth object function. The operator designed with the smoother version has clearly lower amplitude oscillation.
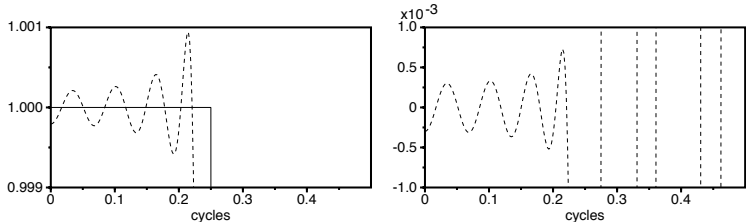

a) standard objective; amplitude (left) and phase error( right).
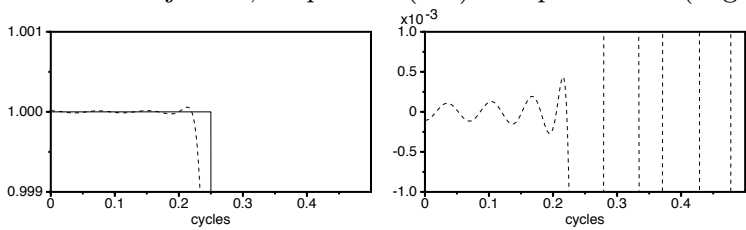

b) smooth objective; amplitude (left) and phase error (right).

Fig. 1: Extrapolation operator spectra for $\frac{\omega}{c}=\frac{k_{N y q}}{2}$. Comparison between WLSQ optimization with $\tilde{\boldsymbol{W}}$ being equal to the phase-shift operator (a) and a smooth version (b) given by equation(5b). The maximum propagation angle is $60^{\circ}$, $\Delta x=10, \Delta z=5$, the operator length is 25 points.

\section{Migration impulse responses}

Zero offset migration experiments are carried out with a 19 points extrapolation operator in a homogeneous medium with a velocity of $2000 \mathrm{~m} / \mathrm{s}$, a receiver length of $2000 \mathrm{~m}$ and a maximum extrapolation depth of $1000 \mathrm{~m}$ with $\Delta x=10, \Delta z=2 \mathrm{~m}$. The zero-offset trace in the middle of the shot record contains three Ricker wavelets at $0.3,0.6$ and $0.9 \mathrm{~s}$, all the other traces are filled with zeros. The source wavelet is sampled with $4 \mathrm{~ms}$ and has a frequency peak at $30 \mathrm{~Hz}$.

Figure 2a shows the impulse responses for a non-recursive reference result. Operators designed with the equiripple approach of the Remez algorithm (Soubaras, 1996) are shown in (b), the WLSQ operators based on the phaseshift operator in (c) and WLSQ operators based on the smooth phase-shift operator in (d). The smooth operator result has on average the least numerical artifacts. However, at the higher angles (above $80^{\circ}$ ) the smooth operator has more and stronger artifacts. In the difference plots of Figure 3 these artifacts are clearly visible. Both WLSQ methods give a better result than the Remez exchange method.

\section{Search for best operators}

The WLSQ operator optimization is done for a fixed operator length, fixed weight factor and a fixed maximum angle of interest. However, convolution operators for lower $k$-values can often be shorter than for higher $k$-values. The weight factor itself has also influence: by choosing a smaller weight outside the wavenumber band of interest a more accurate operator within the desired band will be obtained. By choosing the weight function too small the amplitude can become larger than 1.0 and cause unstable behavior in the recursive algorithm.

Since the WLSQ algorithm used to calculate the opera-

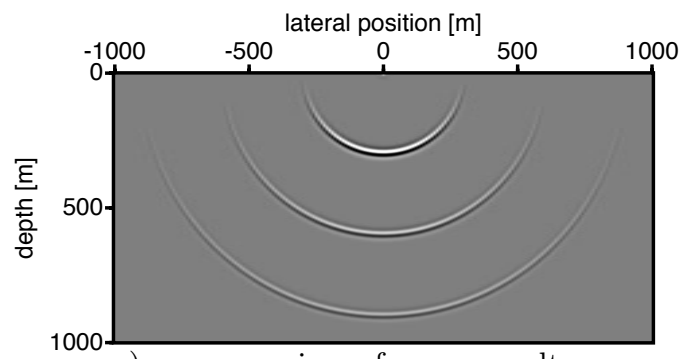

a) non-recursive reference result

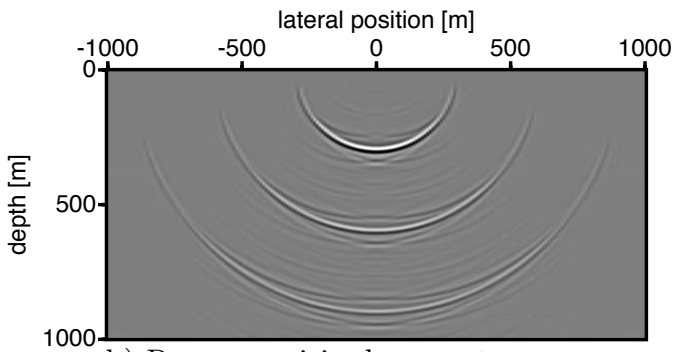

b) Remez equiripple operators

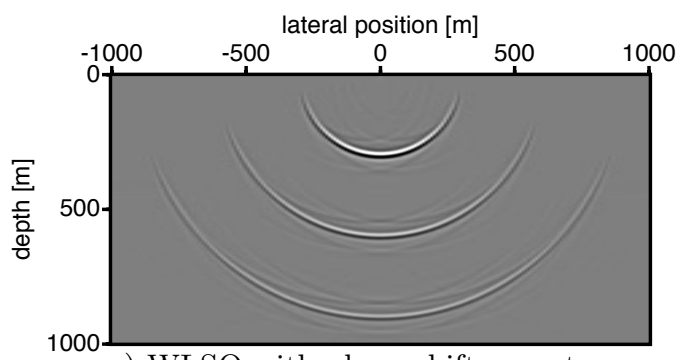

c) WLSQ with phase-shift operators

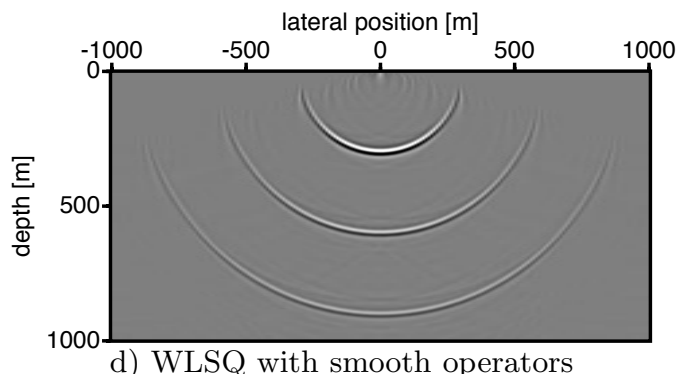

Fig. 2: Migration impulse responses for a non recursive reference result (a) and three recursive methods using: Remez exchange optimized operators (b), WLSQ based on phase-shift operator (c) and WLSQ based on the smooth phase-shift operator (d). The impulse responses are modeled with a frequency range of $(0-80) \mathrm{Hz}, \Delta t=4 \mathrm{~ms}, \Delta x=10 \mathrm{~m}, \Delta z=2 \mathrm{~m}$, a velocity of $2000 \mathrm{~m} / \mathrm{s}$ and an operator length of 19 points. 


\section{Improved extrapolation operator design with the WLSQ method}

tors is very fast (Levinson recursion), it is possible to do a search for the best (shortest, most accurate and stable) operator for a small range of weights and operator sizes in a given wavenumber band. Another advantage of this method is that the error peaks of the used operators will not be aligned anymore for certain wavenumbers and therefore the artifacts will not amplify each other.

The Sigsbee2A data set (Paffenholz, 2002) is depth migrated using one fixed operator length of 25 points, but with a search done for the best operator as function of the weight factor. The pre-stack depth migration result is shown in Figure 4. The steep faults beside the salt are imaged correctly. In the zoom area below the salt, all events that contain reflection energy are visible. Close to the right steep bottom of the salt there are no layers visible and it seems that an internal multiple of the salt body (indicated by an arrow) has been imaged as a steep ghost fault crossing the layers.

The disadvantage of one-way migration is that it is not possible to handle turning/bending waves in the recursive migration (large $>80^{\circ}$ angles of propagation). Also the transmission coefficients are not included resulting in

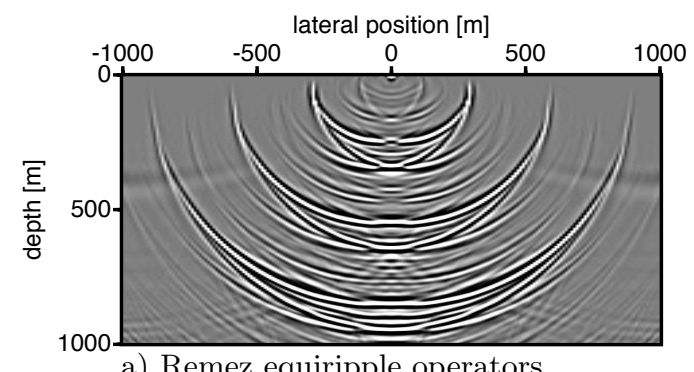

a) Remez equiripple operators

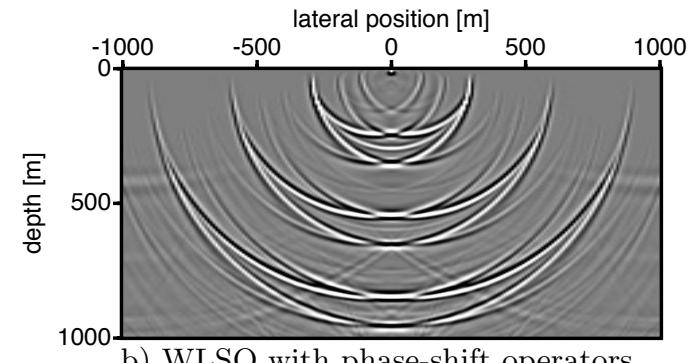

b) WLSQ with phase-shift operators

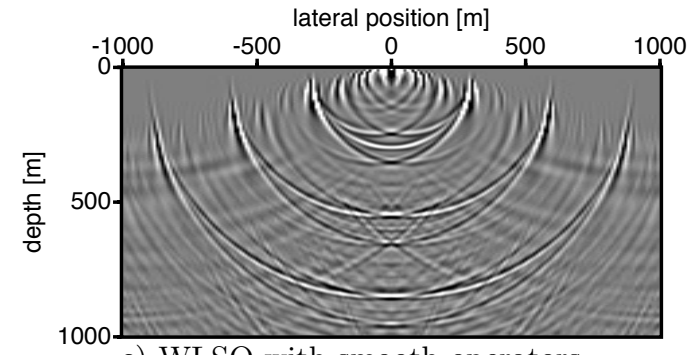

c) WLSQ with smooth operators

Fig. 3: The numerical artifacts of the recursive extrapolation are displayed by subtracting the reference result from the recursive results. Note that the pictures are plotted with a $20 \mathrm{x}$ larger clip value than on Figure 2. lower amplitude, but structural still accurate, images below the salt.

The extension of the WLSQ technique for 2 dimensional operators, to be used in 3 dimensional media is straightforward (Thorbecke and Berkhout, 1994).

\section{Conclusions}

In this abstract the weighted least squares technique has been further improved by using a smooth object function in the estimation of extrapolation operators. The presented results show that these improved operators give very accurate extrapolation results. The WLSQ algorithm used to compute the operators is very fast and multiple evaluations for different weight functions and operator lengths makes it possible to search for the best operator with minimum operator length and smallest amplitude below a certain threshold.

The WLSQ is not only suited for extrapolation operator design, but can also be used in other filter design problems for an efficient and controlled transformation of the (smoothed) operator in the Fourier domain back to a convolution operator in the original domain.

\section{Acknowledgments}

We would like to thank the research school ISES and the Atlass project of the EU for supporting this research.

\section{References}

Blacquiere, G., Debeye, H. W. J., Wapenaar, C. P. A., and Berkhout, A. J. (1989). 3D table-driven migration. Geophys. Prosp., 37(08), 925-958.

Hale, D. (1991). 3-D depth migration via McClellan transformations. Geophysics, 56(11), 1778-1785. Erratum in GEO-57-2-370.

Holberg, O. (1988). Towards optimum one-way wave propagation. Geophys. Prosp., 36(02), 99-114.

Paffenholz, J. e. a. (2002). Sigsbee2a synthetic subsalt data: image quality as function of migration algorithm and velocity model error. In th Annual Internat. Mtg., EAGE, Expanded Abstracts, volume 94.

Nautiyal, A., Gray, S. H., Whitmore, N. D., and Garing, J. D. (1993). Stability versus accuracy for an explicit wavefield extrapolation operator. Geophysics, 58(02), 277-283.

Soubaras, R. (1996). Explicit 3-D migration using equiripple polynomial expansion and Laplacian synthesis. Geophysics, 61(05), 1386-1393.

Thorbecke, J. W. and Berkhout, A. J. (1994). 3-D recursive extrapolation operators: An overview. In 64th Annual Internat. Mtg., Soc. Expl. Geophys., Expanded Abstracts, pages 1262-1265. Soc. Expl. Geophys.

Thorbecke, J. W. and Rietveld, W. E. A. (1994). Optimum extrapolation operators - a comparison. In 56th Mtg. Eur. Assoc. Expl Geophys., Extended Abstracts, page Session:P105. Eur. Assoc. Expl. Geophys. 
Improved extrapolation operator design with the WLSQ method

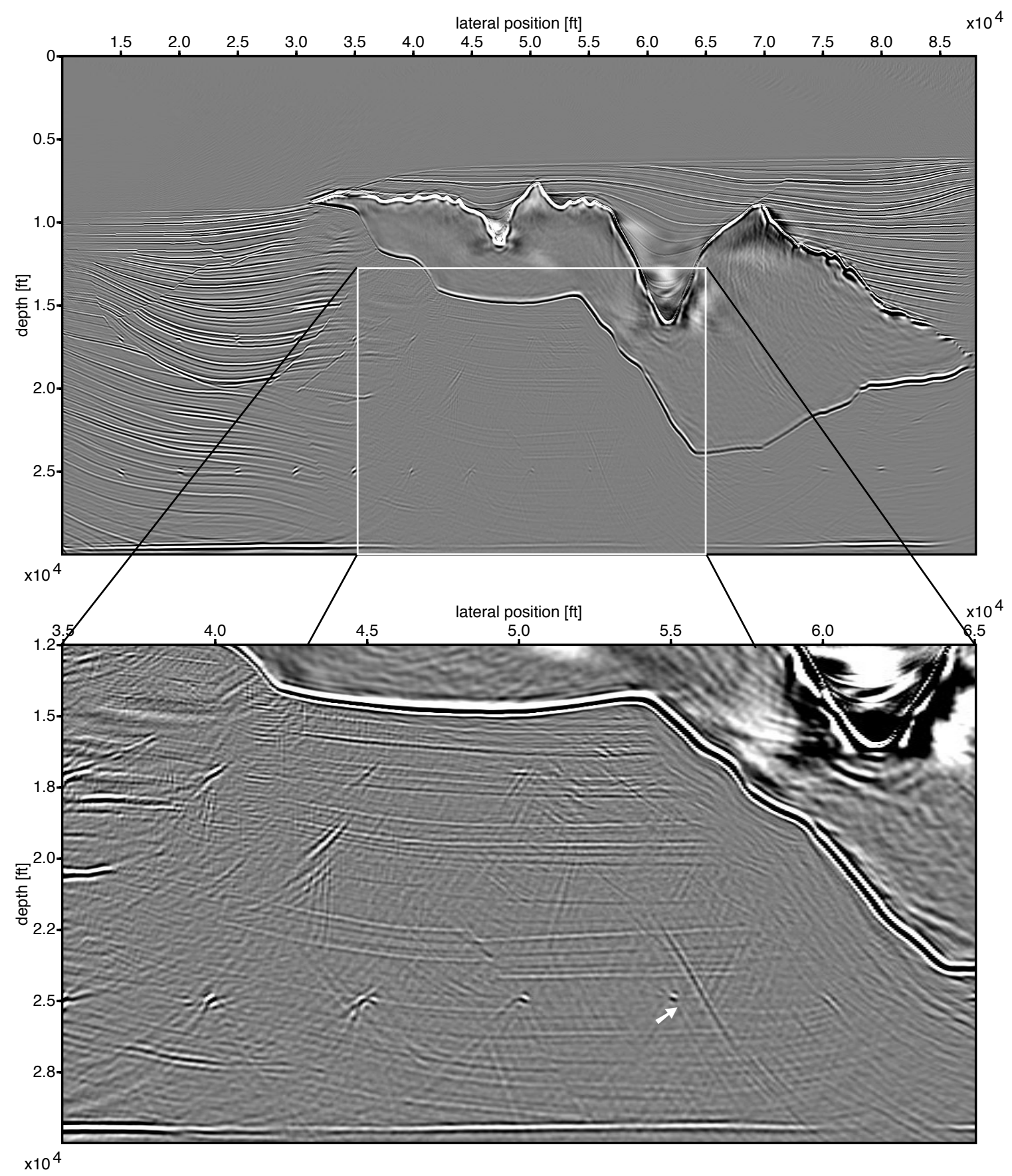

Fig. 4: Pre-stack depth migration of Sigsbee2A data set with optimized WLSQ operators with a fixed length of 25 points. The bottom picture is a zoom of the area below the salt. The transmission coefficients through the salt are not taken into account, giving a lower amplitude image below the salt compared to the surrounding areas. Most events below the salt are imaged, only the turning waves are not imaged correctly. In the zoomed area along the right side of the salt bottom there is a large shadow zone. In this shadow zone only the diffracting point at $(60000,25000)$ is partly imaged. 